\title{
Barriers to Blood Donation Among Mbarara University of Science and Technology Students.
}

\author{
Henry Karugabaa,1,2, Hanifah Nantongo ${ }^{a, 3}$ \\ a Department of Nursing, Mbarara University of Science and Technology
}

\begin{abstract}
\end{abstract}

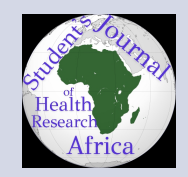

Background: ${ }^{a}$

Blood donation is the voluntary withdrawal of blood from an individual, after undergoing some medical screening in order to ensure the safety of both the donor and the recipient. Blood donation is crucial and indispensable in the medical process of saving lives. Globally, 112.5 million blood donations were made in 180 countries in 2013. Uganda needs at least 340,000 units of safe blood annually, but usually, only 200,000 units are collected yearly. Although massive blood donation campaigns are carried out to obtain blood, there is still more demand for blood within hospitals. Student populations are considered healthy, active, and receptive, thus, regarded as potential blood donors. This study sought to explore the barriers to blood donation among MUST students.

\section{Methodology:}

A qualitative descriptive design was used in this study. A purposive sampling method was used to select respondents from different faculties at MUST. Four FGDs were employed in the study and data were collected using a focus group discussion guide. Inductive content analysis was used to analyze data.

Results:

The study revealed different barriers to blood donation among students. Three broad themes were generated from the analysis; personal barriers related to blood donation, socio-cultural variations affecting blood donation, and the barriers concerning the blood donation process.

\section{Conclusion and recommendations:}

Although participants reported willingness to donate blood, it was found to be affected by barriers ranging from individuals related to the system/process in which blood is collected. Addressing these barriers may improve blood donation by MUST students.

\section{${ }^{a}$ Email: karugabahenry@gmail.com \\ Received: 29th/12/2020 Accepted: \\ 5th/02/2021 Journal of Haematology \\ and Blood transfusion science}

\section{Background:}

Blood transfusion is one of the essential components in the health care management of patients who would be assessed and found to require certain blood products. These blood products can only be obtained through the process of blood donation.

Blood donation is the voluntary withdrawal of blood from an individual, after undergoing some medical screening in order to ensure the safety of both the donor and the recipient of blood including 
its products. The screening includes blood borne diseases such as Hepatitis B, Human Immunodeficiency Virus, and others (Celik, 2015).

Blood donation is crucial and indispensable in the medical process of saving lives (Mohd et al., 2019). Therefore, it is essential to explore the public awareness of blood donation and its eligibility criteria (Lim et al., 2018). The process of blood donation has been regarded as one of our individual and social responsibilities (Celik, 2015) because it being the only source of blood and its products.

Globally, an estimation of 112.5 million blood donations was made in 180 countries during the reporting period of 2013 . The 46 countries in the WHO African Region collected 5.6 million blood donations which were accounting for $4 \%$ of the global donations. Sixty-seven countries reported collecting less than 10 whole blood donations per 1000 populations per year, of which Uganda was inclusive (WHO, 2016). Uganda needs at least 340,000 units of safe blood annually, but usually collects only 200,000 units a year (Global health, 2018). Notably, the collection of sufficient blood to fulfill the demand remains a challenge not only in Uganda but also in other African countries (Ojulong et al., 2016).

Therefore, regular blood donations are important to maintain blood supply and since it may not be stored for a long time (WHO, 2016).

Student populations are considered healthy, active, and receptive, thus, regarded as potential blood donors who meet safe blood donation requirements (Ahmed et al., 2014). Mbarara University of Science and Technology has a high number of students who qualify for blood donations to supply the Mbarara regional blood bank. Although massive blood donation campaigns are carried out to obtain blood to supply health centers, there is still more demand for blood within the hospitals (MUCHAP, 2017). Due to the increase of Trauma, severe malaria, and other commodities like cancers that require massive transfusion.

Mbarara University of Science and Technology is considered a safe haven for blood donations given its student population. Because most of MUST students are healthy young adults that are in a position to donate blood. Mbarara Regional blood bank (MRBB) normally holds blood donation campaigns at this university. However, according to the MRBB daily session reports (2019) concerning blood donation at MUST, an average of 30 phlebotomies a day are obtained which deviates from the daily set target of 50. This study, therefore, sought to explore the barriers to blood donation among students of Mbarara University of Science and Technology.

\section{METHODOLOGY}

\section{Study area}

This study was conducted at Mbarara University of Science and Technology (MUST) which is located in the Southwestern region of Uganda. It's located along the Mbarara-Kabale Highway approximately 267 kilometers from Kampala along Kampala-Kabale Highway in the Mbarara district. MUST have 6 faculties namely; Faculty of Medicine, Faculty of applied sciences and technology, Faculty of business and management sciences, Faculty of computing and informatics sciences, Faculty of interdisciplinary studies, and Faculty of science. MUST was chosen to be the study area because it is one of the institutions with a large student population of 4,431 that could be potential donors.

\section{Study design.}

A qualitative descriptive design was used for this study. This research design was selected because it explained and described the given situation or population. In addition to that, the design is also allowed for the in-depth exploration of issues of interest (Burns \& Grove, 2003).

\section{Study population.}

The study participants were the Mbarara University of Science and Technology students.

\section{Sampling procedures}

A purposive sampling method was used in this study. The purposive sampling method was chosen because it enabled the researcher to identify participants on the basis of those that provided the representation of the general population (Creswell J, 2002) Therefore, participants from all the different faculties were purposively selected.

\section{Sample size determination}

Four FGDs each consisting of 6 participants were used to reach saturation point; whereby no new information is extracted from the newly sampled unit (Burns and Groove, 2003). According to Polit \& Beck (2012), a sample size of 6-12 participants is sufficient as the emphasis is put on the richness of data collected basing on the purpose of the study.

\section{Inclusion criteria}

The study involved MUST students who had consented to participate in the study. 


\section{Exclusion criteria}

The study excluded students who were not around the University at the time of data collection.

\section{Data Collection Tool}

A focus group discussion guide was used as the data collection tool. Discussions were chosen because the study thought to explore the participant's barriers to blood donation which can be answered best by discussions.

\section{Data collection procedure}

Data was collected using an FGD guide. The researcher asked relevant questions and an audio recorder was used to record the participants' responses. Each focused group discussion took about 20-40 minutes and was fully recorded. All members participated actively and they were informed that there was no right and wrong answer. Participants were informed that everyone's opinion was respected and only one participant had to speak at a time. To ensure participants' privacy and confidentiality, discussions were conducted at a convenient time that was suggested by the respondents from their hostels. This created a conducive environment for the participants to narrate their barriers freely without any fears. At the end of the focus group discussion, all participants were thanked and given some refreshments.

\section{Data Analysis}

Data were analyzed using Inductive content analysis by Philipp Mayring (2000). Data collected (audio recordings and researchers' notes) were transcribed (Sutton, 2015). According to Sutton et al., (2015) the steps of analysis for inductive category development as coined by Mayring involve;

- First determining the category definition; the researcher first identified the categories under which he wished to discuss.

- Formulation of the inductive categories or themes out of the material; It's the second step and its where the audio recordings and the researcher's notes were read over and over again to identify the content in them and identify the categories and themes that fit the ones identified in (a) above.

- Revision of the categories and themes and working through the texts. Here the researcher continued to go through the audio recordings and notes over and over again to make sure no content was left out.
- Interpretation of the results. The researcher interpreted and made meaning of the different categories and themes that were identified and discussed the content under these different categories and themes

\section{Rigors of research or trustworthiness}

This was achieved by ensuring trustworthiness or believability. According to Polit et al., (2010), Researchers want their findings to reflect the truth.

To enhance trustworthiness for qualitative research, four criteria were suggested and these included credibility, transferability, dependability, and conformability (Guba and Lincoln, 1985).

\section{Credibility}

This criterion is described as the confidence in the truth of findings as judged by participants and others (Polit\& Beck, 2006). This was achieved by using a purposive sampling procedure, sticking to the inclusion criteria, pre-testing the data collection tool, and prolonged engagement by having sufficient time during the interviews.

\section{Conformability}

According to Polit et al., (2006), conformability is defined as the neutrality of the data or the analysis and interpretation. This was achieved by including narrative quotes into the final research findings.

\section{Dependability}

It refers to the stability of data over time and over conditions (Polit \& Beck, 2006). Dependability was achieved by careful listening to the recorded responses.

\section{Transferability}

This criterion according to Lincoln and Guba (1985) is defined as the extent to which findings can be transferred to other settings. This was achieved through the thick description of the setting and or participants and this encourages the researcher to provide a detailed portrait of the setting in which the research is conducted.

\section{Ethical consideration}

The proposal was submitted to the Faculty Research Committee for approval. An introductory letter was obtained from the Department of nursing and submitted to the dean of students at MUST who permitted the researcher to conduct the study. The study was fully explained to the study participants and they were told that participation in the study was voluntary and that they were free to withdraw at any time they felt like. Participants were also informed that the study findings would only 
be used for the purpose of the study and codes were used on participants to ensure confidentiality.

\section{Dissemination of results}

Results of this study will be compiled into the dissertation and submitted to the FOM at MUST in partial fulfilment for the award of a Bachelor's degree in nursing science. A copy of which will be availed to the Library, Department of Nursing. Another copy will be retained by the researcher.

\section{RESULTS}

\section{Demographic characteristics of the partici- pants.}

The study involved four FGDs each consisting of 6 participants from different faculties of MUST, ten (10) participants were female students while fourteen (14) were male students. The age range of the participants was 21-27. FGD 1 comprised six students from the Faculty of Medicine; FGD 2 comprised six students from the Faculty of Science; FGD 3 consisted of 3 students from the Faculty of Interdisciplinary studies and 3 students from the Faculty of Computing and Informatics; FGD 4 consisted of 3 students from the Faculty of Business Management and 3 students from Faculty of Applied Sciences.

\section{Qualitative results}

Three themes emerged from the study findings as to the barriers to blood donation among students namely; personal barriers related to blood donation, socio-cultural variations, and blood donation, and barriers concerning blood donation as shown in the table below.

\section{THEME ONE: Personal barriers related to blood donation}

There are barriers that were connected to the individual's ability to donate blood. These barriers arose due to side effects, fear for needles, inadequate knowledge, existing health condition, and busy schedules.

\section{Side effects;}

Participants reported that blood donation is associated with various side effects. Some of these related to past experiences like observing their colleagues who fainted after blood. Others speculated that blood donation would affect their lives since a lot of blood was taken. This is also depicted from responses below;

“...... students were seriously collapsing after donating blood and I was there once when I developed this phobia and I felt like not giving blood anymore..." (Male, faculty of medicine, FGD 1)

“..... I fear the side effects from blood donation like the headache, fainting that pulsation is very uncomfortable..." (Male, faculty of interdisciplinary studies, FGD 3)

"...... blood there is someone who donated blood and after donating blood she fainted there you may donate blood and there you just faint..." (Female, faculty of business and management, FGD 4)

One of the FGD participants who had ever donated blood mentioned that the feeling after the donation was not good and this would stop him from donating next time.

"...... the last time I donated I got a lot of side effects like dizziness so it made me uncomfortable..." (Male, Faculty of Science, FGD 2)

\section{Fear for needles;}

Participants expressed a lot of fear for needles whereby some of the participants naturally have a phobia for needles and this stopped them from donating blood. This is depicted in the responses below.

“........ people fear to donate not because they don't have enough blood or what but some people fear to donate because of the horror of the needle, most people fear the needle, me personally I fear it..." (Female, faculty of medicine, FGD 1)

“....... donating blood since it involves pricking like passing needle, to me I have a phobia for needles..." (Male, faculty of science, FGD 2)

Participants feared the pain of the needle and the uncomfortable position on the bed while donating blood and hence this stopped them from donating blood.

“...... I would fear the pain for the needle plus the uncomfortable position on that bed while blood is getting out of my body...." (Male, faculty of interdisciplinary studies, FGD 3)

\section{Inadequate knowledge;}

Participants reported that inadequate knowledge on blood donation and some participant were not aware of the reason of donating and hence this hinders them from donating blood as noted by their excerpts below.

“...... some of us have not realized the role of giving out blood so maybe I'm not informed and this makes me run away from donating so some students don't know the relevancy...."(Male, faculty of interdisciplinary studies, FGD 3) 
Table 1. Themes and categories that emerged from the study

\begin{tabular}{ll}
\hline $\begin{array}{l}\text { Theme } \\
\text { Personal barriers related to } \\
\text { blood donation }\end{array}$ & $\begin{array}{l}\text { Categories } \\
\text { - Side effects } \bullet \text { Busy schedules. } \bullet \text { Existing health condition } \bullet \text { Fear for } \\
\text { Socio-cultural variations and } \\
\text { blood donation }\end{array}$ \\
$\begin{array}{l}\text { Barriers concerning the blood } \\
\text { donation process. }\end{array}$ & $\begin{array}{l}\bullet \text { Inadequate knowledge. } \\
\text { motivation }\end{array}$ \\
\hline
\end{tabular}

“....... students do not know of that reason why they should donate blood.... I think in most cases it is because of the false information about donation that flows....." (Female, faculty of business and management, FGD 4)

“........most people are not sensitized on what the blood is going to be used for and what are the consequences for donating blood... " (Female, faculty of medicine, FGD 1)

\section{Existing health conditions}

Participants reported that existing health condition or disease such as feeling weak, having chronic diseases and terrible diseases like heart diseases, cancer, hypertension, and atherosclerosis hinders them from donating blood. This is depicted in the responses below.

“..... some students who know themselves as sick or weak by having chronic diseases so they will not donate because everyone will think you are putting others at risk....." (Female, faculty of medicine, FGD 3)

“......... there are people with terrible diseases like heart diseases, atherosclerosis, hypertension, cancer, HIV infection so those people will not donate blood......"."(Male, faculty of science, FGD 2)

Participants reported that menstruation periods act as a barrier to blood donation and hence this has stopped them from donating blood during their periods as noted in the excerpt below.

"......... the menstruation periods are a barrier to us women; because they told us when you are in periods do not donate blood so we don't......"(Female, faculty of science, FGD 2)

Participants reported that poor eating habits such as eating junk foods such as Kikomando exposes them to some health conditions and hence stops them from donating blood.

“...... some students have poor nutrition like eating 'Kikomando' for example if I'm not sure of my supper why should I donate blood......" (Male, faculty of science, FGD 2)

\section{Busy schedules}

Participants reported that engagement at schools such as lectures and course works and the mere fact that missing the $80 \%$ attendance would affect their academic status and hence do not get time to donate blood. This was depicted from the responses below.

“............. students are failing to donate blood because of time.......we are always busy we don't have time we have lectures, by the time we finish lectures at 5 pm they have gone....." (Female, faculty of science, FGD 2)

“...... we do various activities like lectures, course works, and many others so you find that we are busy all the time and this limits us from donating...."(Male, faculty of computing science, FGD 3)

“....... people are donating blood and you have a serious lecture and when you miss the $80 \%$ attendance you are gone so that is also a limiting factor...."(Female, faculty of applied sciences, FGD 4)

".... the time those people come to collect blood, they don't look at people's timetables, people don't always get time, you always find them donating when students are in lectures....." (Female, faculty of medicine, FGD 1)

THEME TWO: Socio-cultural variations and blood donation

These are the barriers that arise from the social and cultural aspects in which students live. The theme emerged from two categories which include: religion and culture

\section{Religion}

Participants described that the religion where one belongs for example the Jehovah's Witness followers who don't allow blood transfusion and hence these people cannot donate blood since it is against their belief and thus hinders blood donation as noted below. 
"........ the religion like the Jehovah's Witness followers don't donate blood and neither receive blood so for them it doesn't matter how much you explain to them of the benefits......" (Male, faculty of science, FGD 2)

“..... there are also religion factors, some students belong to those religious groups who don't allow blood donation and this is a barrier for them..." (Male, faculty of computing science, FGD 3)

\section{Culture}

Participants reported that culture is a barrier to blood donation for example in some culture people believe that any bloodshed is a sacrifice to the gods and hence donating blood is worth a sacrifice, some myths are attached to donating blood, and in

In some other cultures, people are scared of their blood being used for ritual purposes.

"..... in our culture, for us we believe that any blood that is shed is the sacrifice to the gods so meaning once you donate your blood into those containers it is worth a sacrifice.....". (Male, faculty of medicine, FGD 1)

"...... the culture I'm coming from we don't donate blood because there are some myths attached to it..." (Female, faculty of interdisciplinary studies, FGD 3)

“......you donate blood... some people can use your samples for ritual purposes, so some students from those family cannot donate their blood..." (Female, faculty of medicine, FGD 1)

One of the FGD Participants reported that in their culture, a person from the royal does not shed blood and hence hindering donating blood as noted in the excerpt below.

“.......in my culture actually we all know that once you belong to the royal family you are not supposed to shade blood, lose blood anyhow like that so for us we don't donate...." (Male, faculty of medicine, FGD 1)

\section{THEME THREE: Barriers concerning the blood} donation process.

These barriers were connected to the way blood donation programs run. These arose due to delays, inconvenient sites, inadequate mobilization, and poor motivation.

\section{Delays;}

Participants reported that delays to receive blood donation cards and blood group results limited them from donating blood. This was depicted from the responses stated below.
“....... I once donated blood they spent actually a full month without bringing back my blood group results not even my card...." (Male, faculty of medicine, FGD 1)

"....... what surprised me they told me to wait to get my blood group but the delay was too much since then I stopped donating blood......" (Female, faculty of science, FGD 2)

The participant reported that the procedure takes a long time and hence in the due course of long waiting made them change their mind hence not donating blood as noted in the excerpt below.

"...... they tell you to wait then you see those procedures they carry out so you find that by the time they call you, you have changed your mind so the long waiting time is not good for me..." (Male, faculty of science, FGD2)

\section{Inconvenient site}

Participants reported that the blood donation site for example lower faculty acted as a barrier to donating blood, whereby they reported that the blood donation team did not find them in their faculties. This is depicted in the responses below.

“........ another barrier when those people come to campus and they just stop in the lower campus actually medicine faculty they do not come to our faculties...."(Female, faculty of science, FGD 2)

Participants reported that the distance from the university to the site of donating blood that is the blood bank hindered them from donating blood as noted in the excerpt below.

"...... those who go donate from the blood bank the distance affects us the university students from donating...." (Female, faculty of science, FGD 2)

\section{Inadequate mobilization}

Participants described that insufficient mobilization i.e. no blood donation campaigns and hence students were less informed about the ongoing blood donation programs thus acting as a barrier to donating blood. This is depicted in the responses below.

"......... by the time I got to know about it, it was done so sometimes we are not informed that they have come..........." (Female, faculty of science, FGD 2)

“.........they don't do campaign because students need to be informed of the purpose for donating blood..." (Male, faculty of computing science, FGD 3)

“..... we lack sensitization...... you are organizing a blood donation activity but how many people know....so information flow is not well-coordinated, 
we are not educated enough....." (Female, faculty of applied sciences, FGD 4)

\section{Poor motivation}

Participants reported that blood donation is associated with poor motivators e.g. a biscuit and a bottle of soda was not so enticing for an adult to donate blood. This is depicted in the responses below.

"......the stimulator which is a soda and a biscuit, for the university people is not so much enticing for someone to put the hand and be pierced moreover in an adult person for a soda and a biscuit..." (Male, faculty of medicine, FGD 1)

“.........those guys actually they do substitute your blood with a simple bottle of soda and a biscuit so you can imagine giving away the whole liter of blood to be substituted with a bottle of soda..." (Female, faculty of business and management sciences, FGD 4)

\section{DISCUSSION}

\section{Introduction:}

This chapter presents the discussion, conclusion, limitation of the study, implications, and recommendations of this study, and areas of future research. The results are discussed in relation to the literature, research question, and conceptual framework. Three main themes emerged from eleven categories that describe the barriers to blood donation among MUST students

\section{Discussion of the results:}

The study revealed that students did not participate in blood donation exercise due to blood donation related side effects like fainting, headache, and dizziness.

The study demonstrated that fear of needles hindered students from donating blood. Participants attributed this to the needle being large in size, causing a lot of pain on pricking which made them uncomfortable. This finding is in line with Ojulong, et al., (2016) who reported that health science students in Namibia expressed a lot of fear of needles which hindered them from donating blood.

Students mentioned that not having adequate knowledge of the relevancy and reasons for blood donation hindered them from donating blood. This was attributed to the insufficient mobilization carried out by the blood collection teams. This finding is congruent with Elias et al., (2016), (salaudeen et al., (2011); Allerson, (2012) whose studies revealed that lack of information or awareness on blood donation was among the main reasons why students never donated blood. Additionally, Demissie, (2014) reported that educating students using simple messages on blood donation should be prioritized in order to fill the information gap.

The study found out that students' existing health conditions impeded them from donating blood. Students attributed this to the fear to worsen their conditions because they had chronic illnesses like diabetes mellitus, HIV/AIDs, heart diseases, atherosclerosis, and cancer. Female students reported that the menstruation period is an obstacle to blood donation. This result is coherent with Tadesse et al., (2018) who indicated that female students in Ethiopia reported that it was bad to donate blood during periods. Similarly, Ojulong, et al., (2016) demonstrated that underweight and feelings of unwellness were barriers to blood donation among learners.

The study identified culture and religion as barriers to blood donation among learners. Participants attributed this to the multiple myths attached to donated blood that it is used for sacrifice and rituals. Some students also reported that the religions they follow do not allow them to donate blood. This finding is coherent with Umeora et al., (2005) where the majority of the respondents believed that their blood was used for witchcraft and that blood donation was against their religious beliefs.

Students' busy schedules have also described a barrier to blood donation. Students attributed this to the countless academic engagements like lectures, course works and that the blood collection teams usually came at inconvenient times for them to donate blood. This finding is in line with Shaz et al., (2010) noted that inconvenient opening time was a barrier to blood donation.

The study demonstrated that the blood donation process is associated with poor motivation to the donors. A bottle of soda and a biscuit was given to the students who donate blood as a reward were unsatisfactory. Furthermore, learners also reported that the blood collection teams delayed issuing out blood donation cards, and blood group results to student donors. 


\section{Conclusion}

The study found personal barriers related to blood donation, socio-cultural variations, and blood donation and blood barriers concerning the blood donation process. However, this study found a unique finding of blood collection teams delaying to issue out blood donation cards and blood group results as a barrier blood donation which is not indicated anywhere in literature.

\section{Limitation of the study}

Students were from MUST only thus the findings cannot represent the barriers by students from other Universities.

\section{Implications and recommendations of the study \\ Education}

The inadequate knowledge of blood donation among students clearly indicates that they are not educated or sensitized about the blood donation process. Therefore, the blood collection team should improve in the way of creating awareness among students concerning blood donation either through education talks and sensitization programs, students should be informed of the relevance of donating blood and the likely side effects faced after donating blood.

\section{Policy:}

The delay in the issuance of blood donation cards and blood group results limits students from donating. It is therefore recommended that policymakers lookout means of issuing out cards earlier and providing immediate feedback of blood group results. Furthermore, the poor motivation i.e. a biscuit and a bottle of soda given after blood donation also limited students from donating. It is therefore recommended that policymakers should improve on the motivating factor to suit students' participation.

\section{Areas of future research:}

Another study should be carried out to identify motivators to blood donation among students

For generalizability, a similar study should be replicated in other blood donation settings

\section{Acknowledgments}

I give special thanks to God Almighty for the gift of life, health, knowledge, and strength in completing this report and the course in general.
This study was a result of cumulative effort from different people and organizations may God bless them abundantly.

In a special way, I would like to acknowledge the profound contributions of my research supervisors Ms. Nantongo Hanifah and Ms. Josephine N Najjuma.

I would like to thank Madam Catherine Atuhaire and all the lecturers in the Department of Nursing at Mbarara University of Science and Technology for their tremendous support towards the success of this research.

I send my dear gratitude to the Kakiiza Charles family for the spiritual, financial, emotional, and social support.

I also acknowledge all my classmates in the BNS and BNC class for all the support and help they rendered to me, May God bless you.

Would like to thank all students at MUST who participated in the study

\section{Abbreviations and Acronyms}

AIDS Acquired Immunodeficiency syndrome

FGD Focus Group Discussion

HIV Human Immunodeficiency Virus

MUCHAP Makerere University Center for Health and Population research.

MUST Mbarara University of Science and Technology

TPB Theory of Planned Behavior

MRBB Mbarara Regional Blood Bank

\section{Definition of Terms}

Barriers: A problem or rule that prevents people from doing something or limits what they can do.

Blood donation: This is a process whereby a person voluntarily has blood drawn and used for transfusions.

Blood: A specialized bodily fluid that delivers necessary nutrients and oxygen.

Donor: An individual who gives out blood.

Phlebotomy: This is a process of making a puncture in the vein with a cannula, for the purpose of taking blood.

Recipient: This is an individual who receives blood.

\section{References}


1. Ahmed, Z., Zafar, M., Ahmed, A.K., Umair, M.A., \& Saddique, M.A. (2014) "Knowledge, Attitude and practices about blood donation among undergraduate medical students in Karachi" Infectious Disease and Therapy, 2(2)https://doi.org/10.4172/2332-0877.1000134

2. Allerson, J. (2012) "Assessment of Selected University Students' Knowledge of Blood Donation and the Relationship with Intent to Donate Blood,"

3. Celik, C., Guven, G., Kan bagisi tutum Olceginin., gelistirilmesi., \& gecerililik Ve guvenirlik Calismasi. (2015) "The development of blood donation attitude scares: reliability and validity study" Erzincan Universities Egitim Fakultesi Dergisi, 17(2): 504-521https://doi.org/10.17556/jef.11163

4. Demissie, A. N. D. B. "Knowledge, attitude and practice on voluntary blood donation and associated factors among Ambo University Regular Students, Ambo Town, Ethiopia," Journal of Community Medicine \& Health Education, 2014; 04(05)https://doi.org/10.4172/2161-0711.1000315

5. Elias, E., Mauka, W., Philemon, N. R., Damian, J. D., Mahande, J. M., \& Msuya, E. S. "Knowledge Attitudes, Practices and Factors associated with voluntary blood donation among University students in Kilimanjaro, Tanzania" Journal of Blood Transfusion, 2016: pg. 6https://doi.org/10.1155/2016/8546803

6. http://www.muchap.org/news/article/09?title=-MUCHAP-raises-230-units-of-blood-in-the-blood-donati on-drive-

7. https://www.theguardian.com/global-development/2018/jan/16/doctors-uganda-warncrisis-level-bloo d-shortage-risks-lives

8. Lim, M.L., Thock, S.H., Andrew, K.G.T., \& Gwee, S.L. (2018) "Determinants of blood donation status in Malaysia: Profiling the non-donors, occasional donors and regular donors" Kajian Malaysia, 36(1): 43-62http s://doi.org/10.21315/km2018.36.1.3

9. Lincoln, Y. S., \& Guba, E. G. (1985) "Naturalistic Inquiry. Newbury Park" CA: Sage Publications.https://doi.o rg/10.1016/0147-1767(85)90062-8

10. Mbarara regional blood bank daily session reports, 2019. (Unpublished)

11. Mohd Asmawi, U. M., Osman, M., Said, N., \& Mohammad, M. (2019). Barriers for blood donation in non-blood donor: a qualitaive study. Environment-Behaviour Proceedings Journal, 4(10), 59-64. https://doi. org/10.21834/e-bpj.v4i10.1622https://doi.org/10.21834/e-bpj.v4i10.1622

12. Ojulong, J., Claude, J.M., \& Monteiro, L. (2016) "Barriers that prevent health science students from donating blood in African setting" International Journal of Community Medicine and Public Health, 3(12): 3544- 3547https://doi.org/10.18203/2394-6040.ijcmph20164289

13. Salaudeen, A.G., \& Odeh, E. (2011) "Knowledge and behavior towards voluntary blood donation among students of a tertiary institution in Nigeria," Nigerian Journal of Clinical Practice, 14 (3): 303-307https://doi.o rg/10.4103/1119-3077.86773

14. Shaz, B. H., \& Hillyer, C.D. (2010) "Minority donation in the United States: challenges and needs" Current Opinion in Hematology, 17: 544-549.https://doi.org/10.1097/MOH.0b013e32833e5ac7

15. Tadesse W, Ayalew Y, Yisma E, Liben ML, \& Wudu M (2018) Knowledge, Attitude, Practice and Associated Factors towards Voluntary Blood Donation among Regular Health Science Students of Samara University, Ethiopia. Health Sci J. Vol. 12 No. 1: 542.https://doi.org/10.21767/1791-809X.1000542

16. Umeora, O., Onuh, S., \& Umeora, M. (2005). Socio-Cultural Barriers to Voluntary Blood Donation for Obstetric Use in a Rural Nigerian Village. African Journal of Reproductive Health / La Revue Africaine De La Santé Reproductive, 9(3), 72-76. doi:10.2307/3583413https://doi.org/10.2307/3583413 